\title{
Magneto-Controlled Enzyme Activity with Locally Produced pH Changes
}

Krisztina Szekeres, ${ }^{\mathrm{a}, \mathrm{b}}$ Paolo Bollella, ${ }^{\text {a,d }}$ Yongwook Kim, ${ }^{\mathrm{c}}$ Sergiy Minko, ${ }^{\mathrm{c}}$ Artem Melman, ${ }^{* a}$ Evgeny Katz*a

${ }^{a}$ Department of Chemistry and Biomolecular Science, Clarkson University, Potsdam, NY 136995810, USA. E-mails: ekatz@clarkson.edu; amelman@clarkson.edu; pbollell@clarkson.edu

${ }^{b}$ Department of Physical Chemistry, Institute of Chemistry, Laboratory of Electrochemistry and Electroanalytical Chemistry, Eötvös Loránd University, Budapest, 1117 Hungary.

c Nanostructured Materials Lab, University of Georgia, Athens, GA 30602, USA

${ }^{d}$ Department of Chemistry, University of Bari A. Moro, Via E. Orabona 4, 70125, Bari, Italy

\section{Supporting Information}

\section{Chemicals and reagents}

Amyloglucosidase from Aspergillus niger (AMG; EC 3.2.1.3), urease from Canavalia ensiformis, Jack bean, (Ur; EC 3.5.1.5), esterase from porcine liver (Est; EC 3.1.1.1), glucose oxidase from Aspergillus niger (GOx; EC 1.1.3.4), peroxidase from horseradish (HRP; EC 1.11.1.7), D(+)maltose monohydrate, urea, ethyl acetate, 2,2'-azino-bis(3-ethylbenzthiazoline-6-sulphonic acid) (ABTS), 1-ethyl-3-(3-dimethylaminopropyl)carbodiimide (EDC), $N$-hydroxysuccinimide (NHS), 2-( $N$-morpholino)ethanesulfonic acid (MES-buffer), 4-(2-hydroxyethyl)-1-piperazineethanesulfonic acid (HEPES-buffer), tetraethyl orthosilicate (TEOS), (3-aminopropyl)triethoxysilane (APTES), $\alpha$-bromoisobutyryl bromide (BIBB), triethylamine (TEA), $N, N, N^{\prime}, N^{\prime \prime}, N^{\prime \prime}$-pentamethyldiethylenetriamine (PMDTA), ascorbic acid (ASCO) and other standard organic and inorganic chemicals, reactants and materials were purchased from MilliporeSigma (formerly Sigma Aldrich). tert-Butyl acrylate (tBA) and poly(ethylene glycol) methyl ether acrylate (PEGMA) with an average M.W of $480 \mathrm{~g} / \mathrm{mol}$ were used after a purification: the inhibitor of tBA and PEGMA was removed using a neutral aluminum oxide column prior to polymerization. Other commercial chemicals listed above were used as supplied without further purification. All experiments were carried out in ultrapure water $(18.2 \mathrm{M} \Omega \cdot \mathrm{cm}$; Barnstead NANOpure Diamond). Preparation and full characterization of surface modified magnetic 
nanoparticles (MNPs) were reported elsewhere ${ }^{1,}$, and a modified procedure is briefly explained below.

\section{Synthesis of silica-coated MNPS}

The MNPs were synthesized using a co-precipitation method as described elsewhere. ${ }^{2}$ Iron chloride salts, $4.43 \mathrm{~g} \mathrm{FeCl}_{3} \bullet 6 \mathrm{H}_{2} \mathrm{O}$ and $1.625 \mathrm{~g} \mathrm{FeCl}_{2} \bullet 4 \mathrm{H}_{2} \mathrm{O}$ were dissolved in $190 \mathrm{~mL}$ of water with a mole ratio of $2 \mathrm{Fe}^{3+}: \mathrm{Fe}^{2+}$ at room temperature $\left(22 \pm 2{ }^{\circ} \mathrm{C}\right)$ under magnetic stirring. Then, $10 \mathrm{~mL}$ of $30 \%$ ammonium hydroxide was added immediately, and then the solution was stirred for additional 10 minutes. Formation of black MNPs was observed. MNPs were collected using a magnet, followed by washing 3 times with water. The surface of MNPs was stabilized with citrate ions by rapid washing with $2 \mathrm{M}$ nitric acid and the addition of $5 \mathrm{~mL}$ of $0.5 \mathrm{M}$ trisodium citrate in water, while maintaining $\mathrm{pH}$ 2.5. After stirring for $1.5 \mathrm{~h}$, MNPs were magnetically isolated, washed with water 2 times, and diluted to $100 \mathrm{~mL}$. The final concentration of MNPs was $2 \%$ wt. The silica-coated MNPs were prepared for further surface modification following Stöber method. ${ }^{3}$ $3 \mathrm{~mL}$ of the MNP stock solution was diluted in a mixture of $160 \mathrm{~mL}$ ethanol and $27 \mathrm{~mL}$ of water in a round bottom flask. Then, $5 \mathrm{~mL}$ of ammonium hydroxide was added. After the colloids were sonicated for $10 \mathrm{~min}$, the colloid-containing flask was placed in an ice bath. While colloids were kept stirring, $500 \mu \mathrm{L}$ of TEOS was added slowly. The reaction proceeded at $0{ }^{\circ} \mathrm{C}$ for $3 \mathrm{~h}$ under magnetic stirring. The reaction was stopped by adding $250 \mu \mathrm{L}$ of $30 \%$ hydrochloric acid. Precipitation of silica-coated MNPs was observed. Precipitation was magnetically collected and washed with water and ethanol 3 times. The final product was stable and dispersed in $80 \mathrm{~mL}$ of ethanol for further modification.

\section{Grafting of PAA-b-PEGMA block-copolymer from the nanoparticles surface}

The PAA- $b$-PEGMA block copolymer was grafted from the surface of the nanoparticles using an activator generated by electron transfer for atom transfer radical polymerization (AGET-ATRP mechanism). ${ }^{4}$ First, ATRP initiator, BIBB, was immobilized on the surface of the silica-coated MNPs. Prepared silica-coated MNPs were dispersed in $80 \mathrm{~mL}$ of ethanol and sonicated for $10 \mathrm{~min}$, followed by the addition of $1 \mathrm{~mL}$ of APTES to introduce amino functional groups at the surface. The reaction was carried out at room temperature for $20 \mathrm{~h}$ under magnetic stirring. The APTES 
immobilized nanoparticles were washed 2 times with ethanol and dichloromethane. The nanoparticles were dispersed in $80 \mathrm{~mL}$ of dry dichloromethane and sonicated for $20 \mathrm{~min}$ to achieve stable dispersion. Afterward, $1.6 \mathrm{~mL}$ of TEA and $800 \mu \mathrm{L}$ of BIBB were added into the dispersion while magnetically stirring. After $1.5 \mathrm{~h}$. nanoparticles were collected using the centrifuge at 12000 rpm for $15 \mathrm{~min}$, and the collected product was washed three times with ethanol. The nanoparticles bearing ATRP initiators were prepared. Polymerization was conducted in two consecutive AGETATRP steps. First, poly(tert-butyl acrylate) (PtBA) was grafted with tBA monomer. Then, the PEGMA block was continuously grafted using the same AGET-ATRP method. Finally, PtBA block was hydrolyzed to convert it into PAA block, bearing carboxylic functional groups for further conjugation of enzymes.

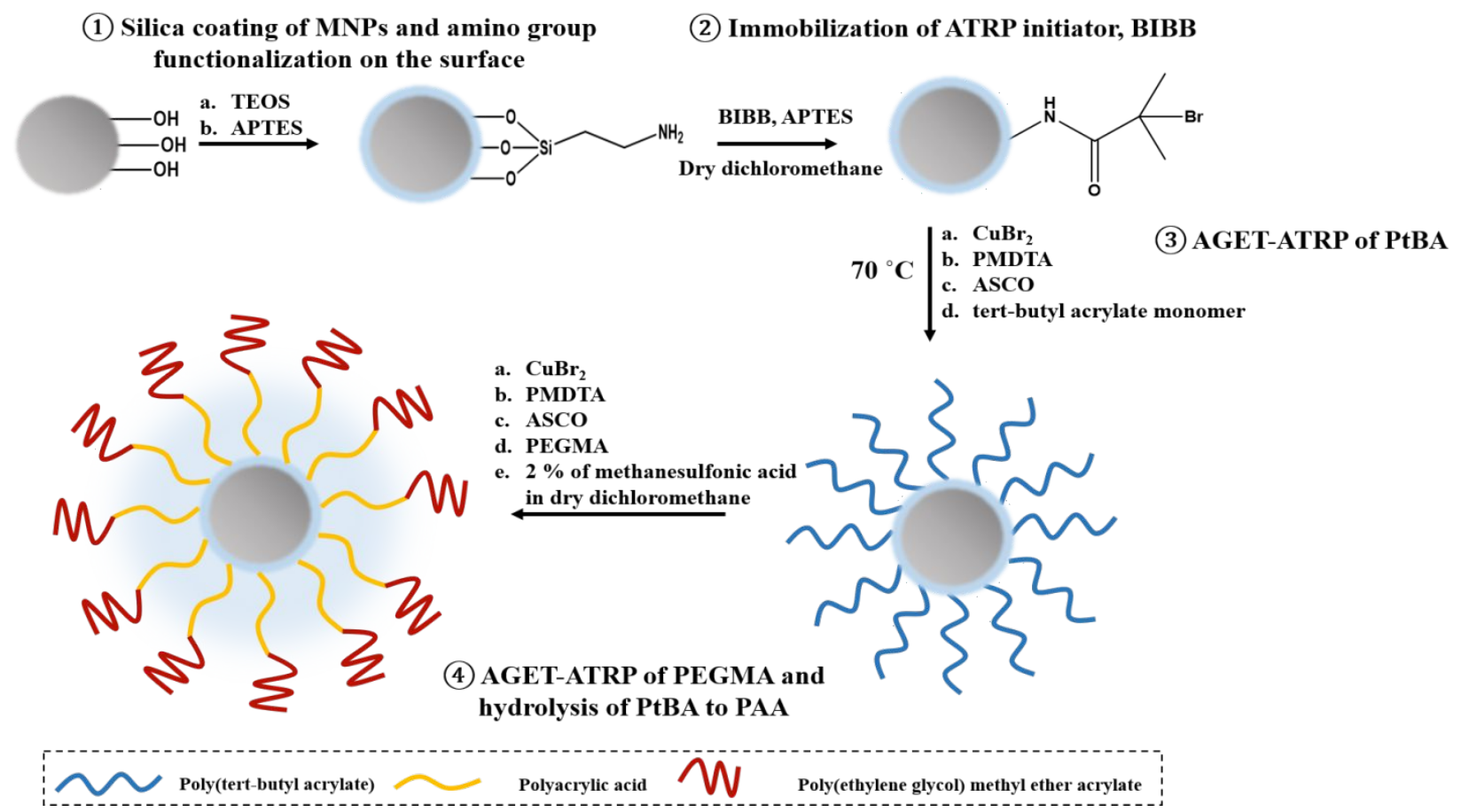

Figure S1. Schematic of modification of MNPs. Detail description is given in sections 2-3.

Polymerization: BIBB immobilized silica-coated MNPs, $210 \mu \mathrm{L}$ of $0.1 \mathrm{M} \mathrm{CuBr}_{2}$, and $320 \mu \mathrm{L}$ of $0.5 \mathrm{M}$ PMDTA were added to $30 \%$ of inhibitor-removed tBA monomer in $45 \mathrm{~mL}$ of ethanol solution. Dissolved oxygen in the solution was removed by purging the solution with argon gas for $20 \mathrm{~min}$. Then, $500 \mu \mathrm{L}$ of ASCO $(0.1762 \mathrm{~g} / \mathrm{mL})$ was dropwise added into the polymerization reactor while degassing with argon. The polymerization proceeded for $30 \mathrm{~min}$ at $70{ }^{\circ} \mathrm{C}$. The 
reaction was stopped by opening the cap and cooling the reactor. The particles were washed with ethanol three times and collected using a centrifuge. Grafting a second PEGMA block was prepared by a similar procedure. $25 \%$ of inhibitor-removed PEGMA in $45 \mathrm{~mL}$ of ethanol solution was polymerized for $1 \mathrm{~h}$ at room temperature. The resulted MNPs were washed with ethanol three times, followed by the conversion of PtBA to PAA in $2 \%$ methanesulfonic acid in $50 \mathrm{~mL}$ of dry dichloromethane for $5 \mathrm{~min}$. After hydrolysis, particles were washed three times with ethanol and dried at $60{ }^{\circ} \mathrm{C}$ in the oven overnight. Powder form of PAA-b-PEGMA grafted silica MNPs were easily dispersed in water and formed stable colloidal status with an average size of $300 \mathrm{~nm}$ and zeta potential $(\zeta)=-30 \mathrm{mV}$ at $\mathrm{pH} 7.4$, characterized by dynamic light scattering (DLS).

Preventing aggregation of the magnetic nanoparticles in the absence of a magnetic field is an extremely important issue in the present project, as well as in some other projects performed by us and reported recently (see ref. 1 in the Supporting Information). The polymer brush bound to the solid core is ended with polyacrylic acid, which provides negative charges at the polymer shell. The negatively charged nanoparticles are electrostatically repulsed from each other, thus preventing their aggregation in the absence of the external magnetic field. Importantly, when the particles are magnetized in the presence of the magnetic field, the magnetic attraction overcomes the electrostatic repulsion, thus resulting in the nanoparticles aggregation. The repulsion (electrostatic) and attraction (magnetic) forces should be optimized to prevent the nanoparticle aggregation in the absence of the magnetic field and allow their aggregation in the presence of the magnetic field. This optimization was performed experimentally.

\section{Covalent functionalization of the MNPs with enzymes (AMG, Ur, Est)}

A suspension of the MNPs (1 mg/mL) in MES-buffer $(50 \mathrm{mM}, \mathrm{pH} 7.4)$ was reacted with $20 \mathrm{mM}$ EDC and $50 \mathrm{mM}$ NHS for $25 \mathrm{~min}$ at room temperature $\left(24 \pm 2{ }^{\circ} \mathrm{C}\right)$. Then, the MNPs were collected by sedimentation with a magnet and the previous reaction solution was replaced with a MESbuffer (50 mM, pH 7.4) containing an enzyme (note that different enzymes were used in preparation of different kinds of the modified MNPs): AMG $(2 \mathrm{kU} / \mathrm{mL})$ or urease $(1 \mathrm{kU} / \mathrm{mL})$ or esterase $(1 \mathrm{kU} / \mathrm{mL})$. The reacting suspension was placed in a shaker for overnight at room temperature. After that, the enzyme functionalized MNPs were collected by sedimentation with a 
magnet, then rinsed three times and re-suspended in $1 \mathrm{~mL}$ HEPES-buffer $(50 \mathrm{mM}, \mathrm{pH} 7)$. The enzyme assay performed before and after the enzyme immobilization demonstrated small decrease of the enzyme activity after the immobilization (ca. $80 \%$ retained activity of the immobilized enzymes). Since the assay of the immobilized enzymes was only possible as the rough estimation, one can conclude that the enzyme activity was almost unaffected by the immobilization procedure. The activity of the enzymes per single magnetic nanoparticle was estimated as: ca. $1.8 \times 10^{-15} \mathrm{U}$ (AMG), ca. $1.7 \times 10^{-15} \mathrm{U}$ (urease), ca. $1.0 \times 10^{-15}$ (esterase). The total amounts of the immobilized enzymes included in the reacting system was approximately $15 \mathrm{mU} / \mathrm{mL}$ (AMG), $11 \mathrm{mU} / \mathrm{mL}$ (urease) or $18 \mathrm{mU} / \mathrm{mL}$ (esterase); optimized experimentally.

\section{Activation and inhibition of AMG bound to the MNPs with urease-functionalized MNPs}

The reaction was performed in $1 \mathrm{~mL} 20 \mathrm{mM}$ maltose solution in a HEPES-buffer, $\mathrm{pH}$ 6.0, using three different buffer concentrations: $1 \mathrm{mM}, 50 \mathrm{mM}$ and $1000 \mathrm{mM}$. The solution also included 2.5 $\mathrm{mM}$ urea, ca. $0.08 \mathrm{mg}$ AMG-functionalized MNPs and ca. $0.08 \mathrm{mg}$ urease-functionalized MNPs. The reaction was performed in the absence or presence of a magnet (OFF or ON magnetic field state, respectively). Two magnets ( $\mathrm{NdFeB}$, grade N42, plating/coating: $\mathrm{Ni}-\mathrm{Cu}-\mathrm{Ni}$, dimensions: $1 / 2 " \times 1 / 4 " \times 1 / 8 "$, B842 from K\&J Magnetics, Inc.) were placed below the reaction tube to create a nearly uniform (homogeneous) magnetic field in the liquid system with the intensity of $0.4 \mathrm{~T}$ measured at the bottom of the reaction tube. The magnetic field was measured with Bell-5180 Gauss/Tesla meter (OECO LLC, Milwaukie, OR, US) with a detachable axial probe that was placed in the immediate proximity of the reaction tube. In the OFF state (absence of the magnet) the MNPs were dispersed in the solution by vigorous mixing. In this state the AMG catalytic activity was high at the bulk $\mathrm{pH}$ 6.0, thus, the reaction resulted in the formation of glucose upon AMG-catalyzed hydrolysis of maltose. In the ON state (presence of the magnet) the MNPs were aggregated and local $\mathrm{pH}$ increased up to ca. $\mathrm{pH} 9$ inhibiting the AMG catalytic activity. The local $\mathrm{pH}$ value was roughly estimated near the aggregated MNPs using $\mathrm{pH}$-sensitive paper-indicator (GE Whatman 10360005, Sigma Aldrich). For this measurements aliquot samples were collected near the magnetic cluster and then applied on the $\mathrm{pH}$-indicator paper. The analysis of the AMG activity was performed in aliquots taken from the main reacting solution containing the enzymemodified magnetic nanoparticles. The aliquots were analyzed in a separate solution. The glucose 
formation was analyzed using standard assay solution composed of GOx (300 U/mL), HRP (300 $\mathrm{U} / \mathrm{mL})$ and ABTS $(25 \mathrm{mM})$ in HEPES-buffer $(10 \mathrm{mM}, \mathrm{pH} 7.0)$. The kinetics of ABTS oxidation was measured at $\lambda=420 \mathrm{~nm}$ using VARIAN CARY Eclipse UV-visible spectrophotometer and used to characterize indirectly the AMG activity. The ON-OFF magnetic field states were repeated several times to analyze reversible dynamic switch of the AMG activity.

The minor absorbance increase (related to a minor AMG activity) in the presence of the magnetic field (Figure 4A in the main paper) originated from the incomplete (but still significant) inhibition of the AMG enzyme. It is not always possible to achieve the complete switch off the enzyme activity - some minor activity might be still present. The present project was not aimed at the total (complete) inhibition of the enzyme, but rather at the demonstration that the enzyme activity can be significantly changed due to local $\mathrm{pH}$ changes triggered by the magnetic signal. It should be noted that many different chemical inhibitors for various enzymes do not completely switch off the enzyme activity. Partial inhibition of enzymes is a common situation. In our specific case the complete inhibition might be achieved upon bigger $\mathrm{pH}$ changes (reaching more basic $\mathrm{pH}$ values). However, this extreme $\mathrm{pH}$ change may result in the irreversible loss of the enzyme activity, which will not allow reversible inhibition/activation of the biocatalytic reaction.

It should be noted, that if the $\mathrm{pH}$-changing enzymatic reaction runs long enough, it can overcome the buffer capacitance, thus resulting in the bulk pH changes. In this case the effect of the $\mathrm{pH}$ change on the AMG activity will be irreversible. In order to prevent this unwanted bulk $\mathrm{pH}$ change the concentration of the substrate (urea for urease or ester for esterase) should be limited. This concentration should be below the limit set by the buffer capacitance, thus the optimized substrate concentration depends on the buffer concentration. In the present preliminary study, we optimized the substrate concentration and the time of the reaction experimentally. In the future studies, the optimization of the system should be performed for different buffer concentrations (capacitance), thus the optimization should include several parameters of the system.

It should be noted that the decrease of the activity of urease and esterase could limit the $\mathrm{pH}$ changes generated by them. Eventually, this might be even better for our system preventing 
formation of extreme $\mathrm{pH}$ values, which can lead to the $\mathrm{AMG}$ irreversible inactivation. We didn't study the $\mathrm{pH}$ dependence of the urease and esterase activity - this was outside the scope of this preliminary study. However, the literature suggests 5,6 that their $\mathrm{pH}$ dependence in the studied range of $\mathrm{pH}$ values is not high and may be neglected for the preliminary study.

In a control experiment magnetic nanoparticles functionalized with AMG were used in the absence of the magnetic nanoparticles modified with esterase or urease. The aggregation and dispersion of the AMG-particles in the presence or absence of the magnetic field did not change the AMG activity (Figure S2).

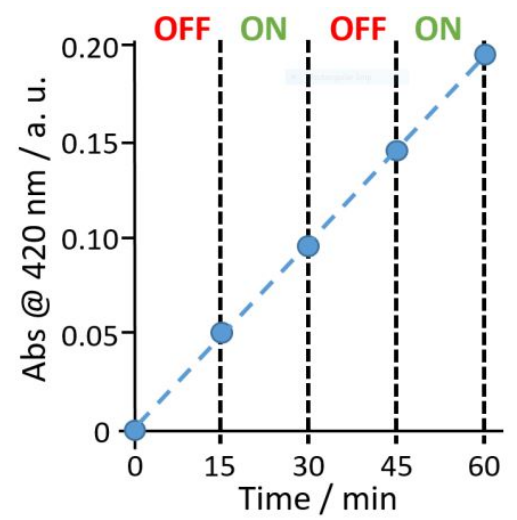

Figure S2. Absorbance changes corresponding to the AMG activity in the absence (OFF) and presence $(\mathrm{ON})$ of the external magnetic field applied on the suspension of the AMGfunctionalized magnetic nanoparticles. In this control experiments the magnetic nanoparticles functionalized with esterase or urease were not added to the system.

\section{Magnetization of the magnetic nanoparticles}



Figure S3. Magnetization function of the nanoparticles. 
In order to aggregate in the presence of the external magnetic field the nanoparticles must be magnetized (note that they are paramagnetic). We characterized the magnetization features of the nanoparticles. ${ }^{1}$ Magnetization of the nanoparticles functionalized with the polymer shell and immobilized enzymes, expressed as the dependence of the magnetic moment vs. magnetic field intensity corresponds to the function expected for the paramagnetic nanospecies. The magnetization reaches saturation at ca. $0.2 \mathrm{~T}$ and the experiments were performed upon applying magnetic field with the intensity of $0.4 \mathrm{~T}$ to ensure the complete (saturated) magnetization of the nanoparticles. The intensity of the applied magnetic field was controlled by the distance between the reaction system and the permanent magnet.

\section{Activation and inhibition of AMG bound to the MNPs with esterase-functionalized MNPs}

The reaction was performed in the same way as described above for the urease-functionalized MNPs with the following difference: the bulk $\mathrm{pH}$ value of the reacting solution was 9.0; the esterase-functionalized MNPs (ca. $0.08 \mathrm{mg}$ ) were used in combination with the AMG-modified MNPs (ca. $0.24 \mathrm{mg})$; the reaction solution included ethyl acetate $(0.25 \mathrm{mM})$ instead of urea.

\section{Characterization of MNPs aggregation by optical microscopy and fluorescent confocal microscopy}

Optical microscope (Nikon) was used to visualize aggregation of the MNPs in the presence of a magnetic field and the formation of $\mathrm{pH}$ gradient around the aggregated MNPs. The formation of the $\mathrm{pH}$ gradient was investigated with a confocal microscope (Leica Confocal Microscope LM6, Leica Microsystems, Buffalo Grove, IL, USA) in presence of 3,4'-dihydroxy-3',5'-bis(dimethylaminomethyl)flavone (FAM345), ${ }^{7}$ the dye with $\mathrm{pH}$-dependent fluorescence ${ }^{8}$ (see the structure below). 


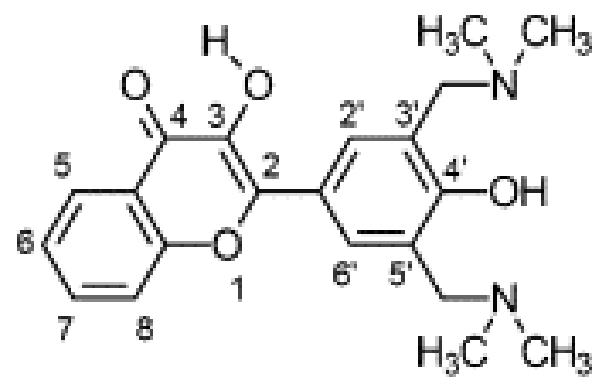

FAM345

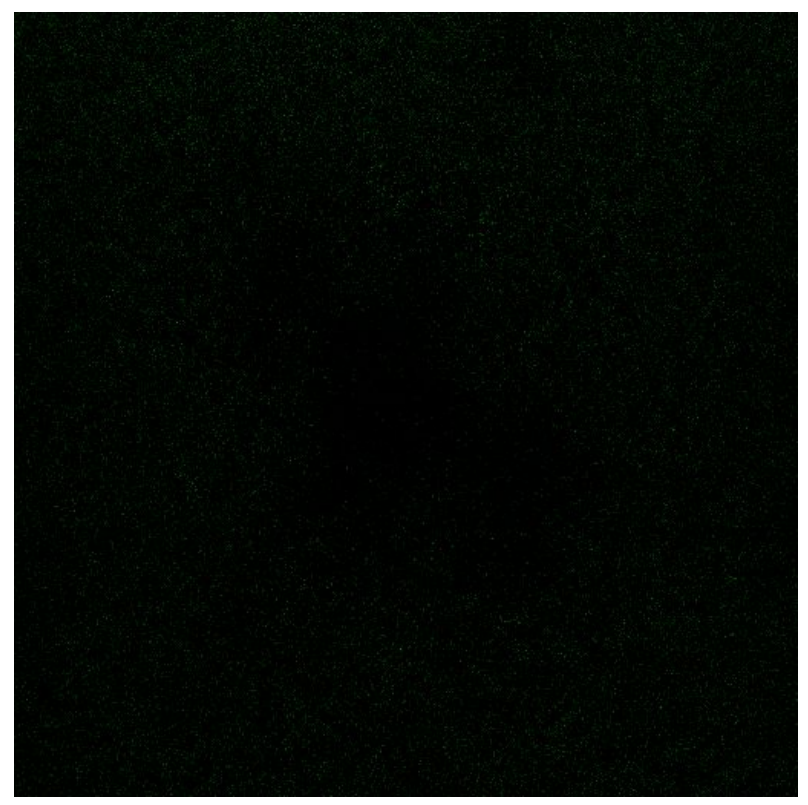

Figure S4. Confocal microscope image showing no fluorescence when measured away from the aggregated nanoparticles in the presence of the magnetic field (compare with Figure 1E that shows intensive green fluorescence around the aggregated nanoparticles).

\section{References}

(1) Zacharchenko, A.; Guz, N.; Laradji, A. M.; Katz, E.; Minko, S. Magnetic field remotely controlled selective biocatalysis. Nature Catal. 2017, 1, 73-81.

(2) Bumb, A.; M. Brechbiel, M. W.; Choyke, P. L; Fugger, L.; Eggeman, A.; Prabhakaran, D.; Hutchinson, J.; Dobson, P. J. Synthesis and characterization of ultra-small superparamagnetic iron oxide nanoparticles thinly coated with silica. Nanotechnology 2008, 19, art No. 335601. 
(3) Deng, Y.-H.; Wang, C.-C.; Hu, J.-H.; Yang, W.-L.; Fu, S.-K. Investigation of formation of silica-coated magnetite nanoparticles via sol-gel approach Colloids Surf. A 2005, 262, 8793.

(4) Jakubowski, W.; Matyjaszewski, K. Activator generated by electron transfer for atom transfer radical polymerization. Macromolecules 2005, 38, 4139-4146.

(5) Krajewska, B.; Ciurli, S. Jack bean (Canavalia ensiformis) urease. Probing acid-base groups of the active site by $\mathrm{pH}$ variation. Plant Physiology and Biochemistry 2005, 43, 651-658.

(6) Bergmann, F.; Segal, R.; Shimoni, A.; Wurzel, M. The pH-dependence of enzymic ester hydrolysis. Biochem. J. 1956, 63, 684-690.

(7) Valuk, V. F.; Duportail, G.; Pivovarenko, V. G. A wide-range fluorescent pH-indicator based on 3-hydroxyflavone structure J. Photochem. Photobiol. A 2005, 175, 226-231.

(8) Fuladpanjeh-Hojaghan, B.; Elsutohy, M. M.; Kabanov, V.; Heyne, B.; Trifkovic, M.; Roberts, E. P. L. In-operando mapping of $\mathrm{pH}$ distribution in electrochemical processes. Angew. Chem. Int. Ed. 2019, 58, 16815-16819. 